\title{
на выставке Securika 2021
}

\author{
Рассказывает руководитель проектного отдела представительства компании TP-Link в России
} А.Ю.Усачёв

DOI: $10.22184 / 2070-8963.2021 .94 .2 .18 .20$

Компания TP-Link, отмечающая в текущем году 25 лет со дня основа-
ния, - один из крупнейших мировых произодителей сетевого оборудо-
вания для малого и среднего бизнеса, которая в первую очередь известна
своими Wi-Fi-роутерами. В последнее время компания завоевала проч-
ные позиции и на рынке корпоративного Wi-Fi, а также в этом году пред-
ставляет новую линейку решений для видеонаблюдения под брендом VIGI.
В преддверии выставки Sесuгіka 2021 корреспондент "ПЕРвОЙ МИлИ" взял
краткое интервью у руководителя проектного отдела представительства
ТР-Link в России Алексея Усачёва.

В 2021 году TP-Link отмечает четвертьвековой юбилей. Что компания представляет собой сегодня?

Пройдя долгий путь с момента выпуска первой сетевой карты TP-Link со скоростью в 10 Мбит/с в 1996 году до сегодняшних дней, когда мы стали лидером в сегменте беспроводного сетевого оборудования для дома и малого и среднего бизнеса, нам действительно есть что вспомнить. Важными вехами стали 2005 год, когда компания впервые вышла на международный рынок, а также 2008 и 2009-й, когда последовательно были открыты офисы в США, Германии и России.

Сегодня мы поставляем оборудование более чем в 170 стран и обеспечиваем доступ в сеть и интернет миллиардам людей во всем мире. У нашей компании 44 региональных офиса, которые способствуют развитию бизнеса, обеспечивают клиентскую поддержку, логистику и маркетинг.

В 2021 году TP-Link обладает пятью собственными заводами, которые позволяют компании производить сетевое оборудование для домашнего и бизнес-сегмента, в том числе обеспечивая потребности крупных системных интеграторов и интернетпровайдеров, а также конечных пользователей, которые стремятся сделать свои дома умнее и технологичнее.

Наша компания является постоянным членом Wi-Fi Alliance и одной из первых, кто начинает внедрять и делать массовыми новейшие разработки в области потребительской беспроводной электроники. Новинки нашей продукции ежегодно представлены на международных выставках CES, MWC и IFA, а партнерство с Qualcomm, Broadcom, Intel и MediaTek позволяет нам создавать актуальные и востребованные рынком сетевые решения.

Какие направления бизнеса являются приоритетными для компании на российском рынке?

В прошлом году TP-Link удалось продвинуться по части популяризации решений, связанных с технологиями Wi-Fi 6, Mesh и бесшовного роуминга, и в этом году мы планируем продолжить работу в этом направлении. В значительной степени этому поспособствовал запуск обновленной линейки корпоративного беспроводного оборудования под бредом TP-Link Omada. Оборудование с использованием Wi-Fi 6 и Mesh уже доступно как конечным пользователям, так и корпоративным заказчикам. Обеспечение быстрой и качественной передачи информации посредством беспроводного доступа - это постоянный приоритет для нашей компании, и большая часть 
флагманских решений будет направлена на удовлетворение именно этой потребности современных клиентов.

В текущем месяце мы представляем в России новую линейку оборудования для профессионального видеонаблюдения - TP-Link VIGI. C развитием Интернета вещей и умных городов мы понимаем востребованность современных рентабельных решений, которые призваны сделать безопаснее как бизнес, так и социальную среду.

Вместе с этим мы видим большой потенциал в сотрудничестве с крупными локальными IT-компаниями по внедрению технологий искусственного интеллекта (ИИ) в некоторые из наших потребительских продуктов, мы, безусловно, видим важность удаленного управления масштабной сетевой инфраструктурой в постковидную эпоху, а также осознаем, что доля пользователей мобильного широкополосного доступа в интернет растет ежегодно.

Что предлагается TP-Link для сектора В2В?

TP-Link обладает широким портфелем сетевых решений, которые способны удовлетворить потребности заказчиков из самых различных сфер. Если говорить про корпоративный Wi-Fi и коммутацию, то сетевая инфраструктура, созданная на TP-Link, уже применяется предприятиями из областей отельного бизнеса, образования, медицины, розничной торговли и логистики. Устройства TP-Link выбирают клиенты из различных отраслей, частные и государственные организации.

Мы обеспечиваем доступ к Wi-Fi на крупных международных мероприятиях и выставках, в студенческих городках, на объектах транспортной инфраструктуры и в поездах дальнего следования, гостиничных комплексах и курортах по всему миру. С этого года мы будем активно развивать линейку устройств профессионального видеонаблюдения и будем рады предложить текущим и новым клиентам интересные и рентабельные решения на их основе.

Российское представительство TP-Link оказывает поддержку заказчикам при подборе проектного оборудования, помогает с радиопланированием, а также совместно с инженерами в Китае трудится над доработкой и кастомизацией продукта под различные требования клиентов.

В прошлом году компания также сосредоточилась на работе с российскими операторами связи различного масштаба. Некоторое время назад мы представили комплексное решение, способствующее снижению операционных затрат у провайдеров-TP-Link Agile. Оно включает ACS-сервер для уда-
Преимущество программноконфигурируемой сети над традиционным подходом очевидно. Сеть класса SDN позволяет производить настройку, управление и мониторинг сетевой инфраструктуры на множестве удаленных объектов централизованно и, благодаря облачному сервису, везде, где есть доступ в интернет. Сегодня, когда многие заказчики стремятся обеспечить дополнительную экономическую эффективность для бизнеса и трудовых ресурсов, а также обеспечить безопасность сотрудников, такие решения становятся особенно актуальными.

TP-Link Omada SDN - это программно-конфигурируемая сетевая инфраструктура, которая включает в себя точки доступа, коммутаторы и шлюзы, а также предоставляет удобное облачное управление сетевыми устройствами на множестве объектов из веб-интерфейса или мобиль-

\section{В текущем месяце мы представляем в России новую линейку оборудования для профессионального видеонаблюдения}

ленного управления абонентскими устройствами и повышения эффективности поддержки конечных пользователей, а также решение Agile Config, которое позволяет провайдерам самостоятельно кастомизировать эти устройства под внутренние настройки сети.

Расскажите подробнее о решении Omada SDN. Каковы преимущества программно-конфигурируемой сети над традиционным подходом? ного приложения. Если до SDN требовалось настраивать каждое сетевое устройство или точку доступа по отдельности, то при использовании SDN возможно централизованно назначить настройки и управлять всеми устройствами, используя единый контроллер. TP-Link Omada SDN позволяет удаленно настраивать устройства и управлять ими в каждой локации без необходимости отправки инженера на объекты, что повышает эффективность сети 
и снижает затраты на ее развертывание, работу и обслуживание.

Запуск инфраструктуры Omada $\mathrm{SDN}$ сопровождался одновременным выводом на рынок точек доступа TP-Link EAP HD с поддержкой Wi-Fi 6, которые призваны обеспечить более высокую производительность беспроводного доступа в интернет при подключении большого числа пользователей, потребляющих значительные объемы контента.

B TP-Link Omada SDN предусмотрены два вида контроллеров - облачный и гибридный аппаратный (устанавливаемый локально, но с полным доступом к облаку), которые обеспечивают полный контроль над сетью. Контроллер Omada SDN автоматически настраивает параметры оборудования, упрощая процесс развертывания сети, а также обеспечивает централизованное управление на множестве объектов. У заказчика есть выбор: управлять сетью удаленно или поручить это локальному сотруднику.

Решение Omada SDN использует три ключевые технологии, которые позволяют быстро и удобно управлять масштабной сетью. В первую очередь это технология на базе ИИ, которая обеспечивает эффективную и производительную работу $\mathrm{Wi}$-Fi, изменяя настройки каналов и мощности передатчика для соседних точек доступа в одной сети. Она также анализирует возможные проблемы в сети, оповещает пользователей и предлагает решения. Пользователю придется меньше беспокоиться об интерференции беспроводного сигнала между соседними точками доступа, а также о возможных сбоях в работе сети.

Второй ключевой технологией является Zero-Touch Provisioning (автоматическая настройка сетевых параметров). Рассмотрим схему, когда клиенты используют услуги системных интеграторов.
После подключения устройств к локальной сети и сети питания системный интегратор получает возможность управлять ими с помощью облачного контроллера, в том числе отправлять файлы настроек. Таким образом, устройства могут настраиваться коллективно и на удалении от объекта. Настройка не требует глубоких технических знаний, что позволит заказчику снизить расходы.

Третья технология - возможность развертывания эффективной сети Wi-Fi в среде с высоким числом клиентов. Благодаря чипсетам высокого класса, выделенным антеннам, автоматическому выбору канала и настройке мощности передатчика точки доступа Omada с поддержкой Wi-Fi 5 и Wi-Fi 6 обеспечат высокую производительность в загруженной пользовательской среде.

Какие новинки TP-Link представляет для видеонаблюдения?

Новая линейка оборудования компании для видеонаблюдения называется TP-Link VIGI (от английского vigilance - бдительность), и ее задача - предоставить малым и средним предприятиям удобные, надежные и понятные решения для защиты бизнеса. Поскольку бдительность подразумевает внимательность к деталям и наблюдательность, это название также отражает наш профессиональный подход к безопасности.

Устройства VIGI нацелены на различные сценарии, которые позволят владельцам бизнеса защитить свои вложения. Широкая функциональность устройств, высокое качество сборки и простая инсталляция - все это обеспечит дополнительную стабильность в ведении операционной деятельности.

Главные принципы, которыми TP-Link руководствовалась при разработке продуктовой линейки - это важность защиты от рисков (потому что мы понимаем, насколько ведение малого и среднего бизнеса может быть сопряжено с рисками), надежность (мы знаем, насколько от надежности и отказоустойчивости оборудования зависит без опасность предприятия), а также профессионализм (мы понимаем, насколько для профессионального оборудования важно наличие продвинутых функций).

На старте в России линейка TP-Link VIGI получит камеру наружного наблюдения и турельную камеру для помещений, а также восьмиканальный сетевой видеорегистратор с возможностью удаленного мониторинга и собственным интерфейсом для управления видеокамерами, записи видеопотока и последующего просмотра этих записей. Базовые функции камер включают в себя возможность просмотра видео в разрешении 3 МП в формате Н.264+, интеллектуальное обнаружение движе ния, инфракрасную подсветку для видеонаблюдения в ночное время, широкий динамический диапазон видео, шумопо давление, а также по части коммутации и питания - поддержку PoЕ. Сетевой видеорегистратор позволит хранить и воспроизводить видеопоток с подключенных камер, подключать жесткий диск объемом до 10 ТБ и сохранять видео продолжительностью до 720 дней. Мобильное приложение VIGI обеспечит доступ к сетевому видеорегистратору из смартфона в любом месте, где есть доступ к интернету.

В ближайшем будущем TP-Link также представит новые модели камер в линейке VIGI, среди которых будут купольные и панорамные, поворотные камеры для улицы, с возможностью сетевого подключения как по кабелю, так и по Wi-Fi.

Спасибо за интересную беседу. Записал С.А.Попов. 


\section{tp-link | VIGI}

\section{Видеонаблюдение от TP-Link}

\section{Профессиональная линейка CCTV для бизнеса}

Камеры | Сетевые видеорегистраторы |Удалённый мониторинг

\section{Камеры VIGI}

- HD Высокое разрешение (ЗМП)

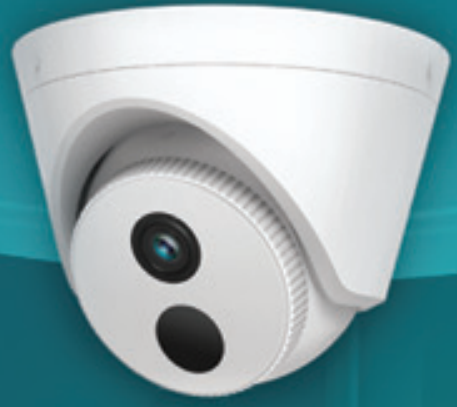

룰. Интеллектуальное

Широкий динамический

wDR диапазон

IR) умная ИК-подсветка

$\overline{\text { DNR }}$ Шумоподавление 3D DNR

() POE/12 B

Iiil постоянный ток
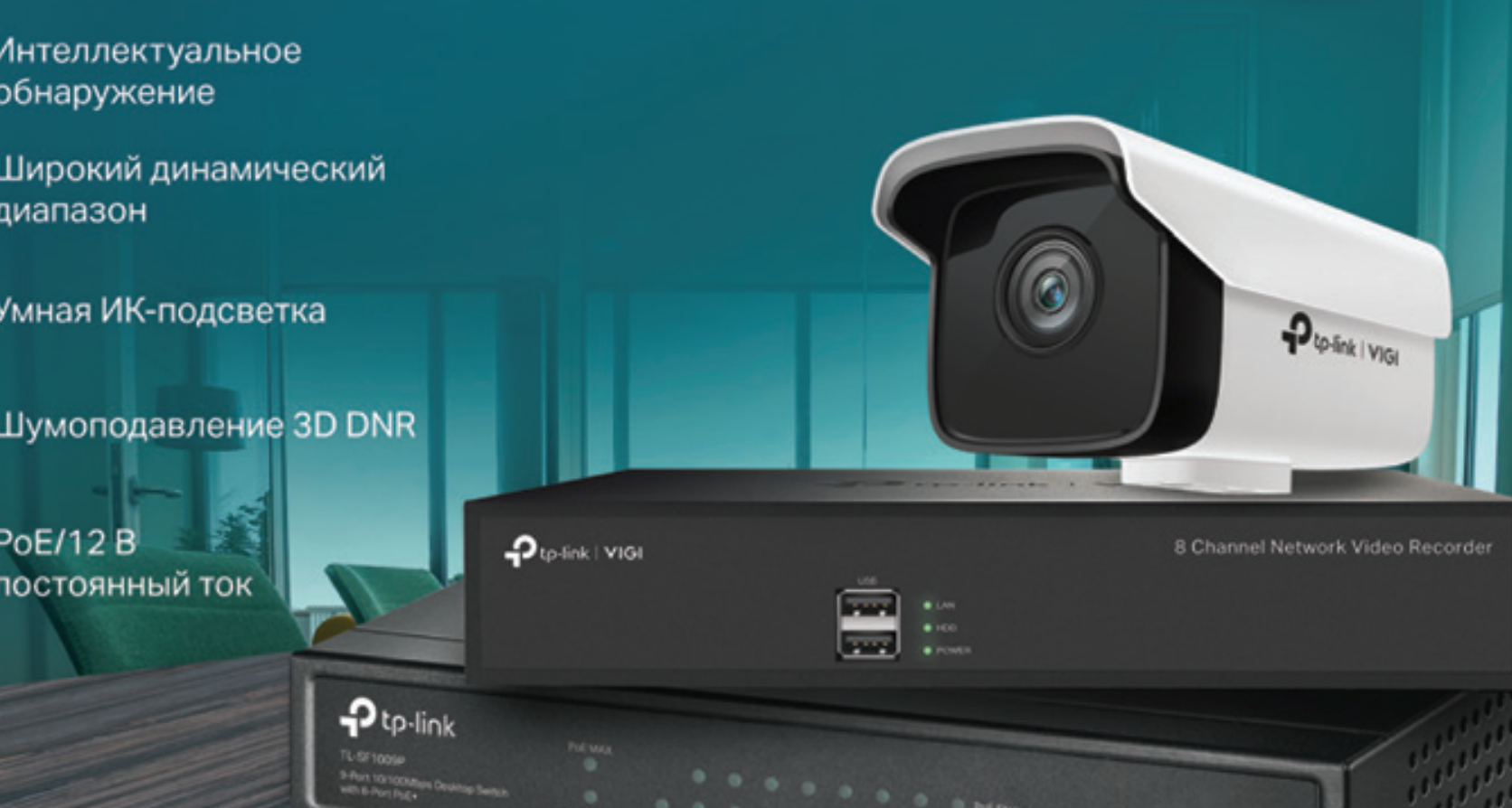

\section{Видеорегистраторы VIGI}

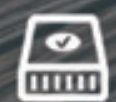

Поддержка HDD до 10 ТБ

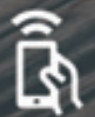

Удалённый мониторинг
Двусторонняя аудиосвязь

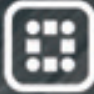

Стандарт ONVIF
H다임

HDMI/VGA

HDD до 10 мб мониторинг аудиосвязь ONVIF

способность
до $80 \mathrm{M6nT/C}$

KOHТАКТЫ:

Тел.: +7 (495) 2285566 | E-mail: project.ru@tp-link.com | tp-link.com/ru/business-networking/ 